\title{
Slit-miR-218-Robo axis regulates retinal neovascularization
}

\author{
YICHUN KONG ${ }^{1-4^{*}}$, BEI SUN ${ }^{5,6^{*}}$, QUANHONG HAN ${ }^{1-4}$, SHUANG HAN ${ }^{1-4}$, \\ YUCHUAN WANG ${ }^{1-4}$ and YING CHEN ${ }^{1-4}$
}

\begin{abstract}
${ }^{1}$ Tianjin Eye Hospital, ${ }^{2}$ Tianjin Key Lab of Ophthalmology and Visual Science, and ${ }^{3}$ Tianjin Eye Institute, Heping, Tianjin 300020; ${ }^{4}$ Clinical College of Ophthalmology, Tianjin Medical University, ${ }^{5}$ Key Laboratory of Hormones and Development, Ministry of Health, and ${ }^{6}$ Metabolic Diseases Hospital and Tianjin Institute of Endocrinology, Tianjin Medical University, Heping, Tianjin 300070, P.R. China
\end{abstract}

Received July 31, 2015; Accepted February 19, 2016

DOI: $10.3892 / \mathrm{ijmm} .2016 .2511$

\begin{abstract}
R-218 is an important intronic microRNA (miRNA or miR) which is known to regulate angiogenesis in tumors. The present study aimed to investigate the effects of miR-218, as well as its host genes, Slit2 and Slit3, on oxygen-induced retinal neovascularization (RNV) and to explore the associated mechanisms of action. For this purpose, a mouse model of oxygen-induced retinopathy (OIR) was established. The expression levels of miR-218-1 and miR-218-2, as well as those of their host genes, Slit2 and Slit3, were determined by RT-qPCR. Fluorescein angiography was performed on the retinas of the mice with OIR, and RNV was quantified by H\&E staining in order to evaluate the effect of pCDH-CMV-miR-218 intravitreal injection on RNV in the mouse model of OIR. Roundabout, axon guidance receptor, homolog 1 (Robo1) expression was detected in mouse retinal vascular endothelial cells expressing high or low levels of miR-218 and in retinal tissues from mice with OIR by western blot analysis. Cell migration was evaluated by a scratch wound assay. We noted that in the mice with OIR, the expression level of miR-218 was significantly downregulated. We also noted that Robol expression was suppressed by miR-218. Furthermore, in the mice with OIR, the expression level of miR-218 was significantly downregulated, and that of miR-218-1 and its host gene, Slit2, was concomitantly downregulated as well. The restoration of miR-218 inhibited retinal angiogenesis by targeting Robol. Taken together, our findings suggest that the Slit2-miR-218-Robol axis contributes to the inhibition of retinal angiogenesis and that miR-218 may be a new therapeutic target for preventing RNV.
\end{abstract}

Correspondence to: Dr Yichun Kong, Tianjin Eye Hospital, 4 Gansu Road, Heping, Tianjin 300020, P.R. China

E-mail:kongyc1942@yahoo.com

*Contributed equally

Key words: retinal neovascularization, oxygen-induced retinopathy, miR-218, Slit2, Slit3, Robo1

\section{Introduction}

Retinal neovascularization (RNV) is the growth of new capillaries sprouting from the retinal veins and extending along the vitreous surface of the retina, which leads to vitreous hemorrhage, retinal detachment and even blindness (1-4). RNV plays an important role in many ocular diseases, such as proliferative diabetic retinopathy (PDR), retinal vein occlusion and retinopathy of prematurity (ROP). However, the pathological mechanisms involved in the formation and development of RNV remain unknown and still need to be further studied.

Slit-Robo signaling consists of three Slit (Slit1-3) secreted proteins and their four corresponding receptors [roundabout, axon guidance receptor, homologs 1-4 (Robo1-4)]. Slit-Robo signaling was first noted as acting as a repulsive cue in axonal guidance $(5,6)$. Subsequently, the roles of Slit-Robo in organogenesis (7), neuronal migration (8) and tumor development (9-12) have been explored. Great progress has been made in studying the role of Robol signaling in angiogenesis, but the role of Robol in angiogenesis is still not fully understood. Han and Zhang demonstrated that Robol is involved in inhibiting corneal neovascularization (13). However, there is also evidence that Robol is pro-angiogenic: Wang et al (14) have reported that Robol mediates human umbilical vein endothelial cell (HUVEC) migration upon Slit2 stimulation, indicating that Slit2-Robo1 signaling promotes cancer angiogenesis. A previous study by Zhou et al (15) found that Robol was expressed in fibrovascular membranes (FVMs), demonstrating that Robol contributes to the development of diabetic retinopathy. The role of Slit-Robo signaling in RNV of oxygeninduced retinopathy (OIR) is researched in the present study.

MicroRNAs (miRNAs or miRs), small non-coding RNAs which are 21-24 nucleotides in length, act as important regulators of gene expression in mammals; several mammalian miRNAs have been identified as being located in the intronic regions of protein-encoding genes (host genes), termed intronic miRNAs (16). Intronic miRNAs can modulate the function of their host gene as the majority of them are coordinately expressed with the host genes $(17,18)$. One example of endogenous RNA interference is when miRNAs negatively regulate gene translation by pairing with the 3'-UTR of a specific mRNA (19), and are involved in many functional regulatory processes (20-22). 
Many miRNAs have been found to play key roles in ocular neovascularization. Intraocular injection of certain miRNAs, such as miR-31 (21), miR-150 (23), miR-184 (23), miR-126 (24) and miR-410, has been shown to significantly inhibit retinal and choroidal neovascularization (25). miRNA-218 is an important intronic miRNA encoded by the Slit 2 and Slit3 genes, which directly represses the expression of Robo1, Robo2 and contributes to retinal angiogenesis in mouse embryos (26). In order to investigate the effects of miR-218 on oxygen-induced RNV, a mouse model of OIR was established in the present study.

\section{Materials and methods}

Cell culture and transfection. Mouse retinal vascular endothelial cells were purchased from PriCells Biomedical Technology Co., Ltd. (Wuhan, China), and were cultured in DMEM supplemented with $10 \%$ heat-inactivated fetal bovine serum, $100 \mathrm{U} / \mathrm{ml}$ penicillin, and $100 \mathrm{mg} / 1$ streptomycin (all from Gibco Life Technologies, Carlsbad, CA, USA) under conditions with $5 \% \mathrm{CO}_{2}$, at $37^{\circ} \mathrm{C}$, in a humidified incubator.

Cells in the exponential growth phase were seeded in 6-well plates (Corning Incorporated, Corning, NY, USA) at a density of $5 \times 10^{4}$ cells $/ \mathrm{ml}$. The cells were transfected with miR-218 mimic, miR-218 inhibitor, and the negative control (all from GenePharma, Shanghai, China) using Lipofectamine 2000 (Invitrogen, Carlsbad, CA, USA) at a 5:1 volume/mass ratio of reagent to oligodeoxynucleotide in serum-free M199 for $6 \mathrm{~h}$. After transfection, the cells were incubated in complete DMEM. Transfection of the miR-218 mimics, miR-218 inhibitor and the negative control was performed using $1.0 \mu \mathrm{g}$ of DNA per transfection. All transfections were performed according to the manufacturer's instructions.

siRNA design. Robo1 siRNA (sense, 5'-CGGGAAAGGCCG AGGAACAAAGGCAGC-3' and antisense, 5'-GCUGCCUUU GUUCCUCGGCCUUUCCCG-3') was synthesized by Guangzhou RiboBio Co., Ltd. (Guangzhou, China).

Plasmid construction. miR-218 was amplified using the following primers: miR-218 forward, 5'-TTCTGAGGATCC GTGGAGGCACCTTTTCCATA-3' and reverse, 5'-ATTCTA AGATCTTTCACAGCTAGTCACACAATGG-3'. Plasmid vector $\mathrm{pCDH}-\mathrm{CMV}-\mathrm{miR}-218$ was constructed by inserting a pri-miR-218 PCR fragment into the plasmid vector pCDH-CMV-MCS-EF1-Puro (Spiral Biotech, Inc., Norwood, MA, USA) through EcoRI and NotI digestion. The pCDH-CMVmock vector was used as the negative control.

Reverse transcription-quantitative PCR (RT-qPCR). Total RNA was extracted from cells and retinal tissues using TRIzol reagent (Invitrogen). cDNA was synthesized, according to the manufacturer's instructions, with a cDNA synthesis kit (Takara Bio, Inc., Otsu, Japan). qPCR was undertaken using SYBR-Green.

The primer sequences were as follows: miR-218, 5'-TTG TGCTTGATCTAACCATGT-3'; miR-218-1, 5'-CCATGGAA CGTCACGCAGC-3'; miR-218-2, 5'-GCGGAAAGCACC GTGCTC-3'; Slit2 forward, 5'-GCGCGTCTGGTGTGAAT GAA-3' and reverse, 5'-CACAGTGGC ACCAGGAGCAT-3'; Slit3 forward, 5'-TGGAAATACGCCTAGAACAG-3' and reverse, 5'-ACCAGCGACGTGAGTGAT-3'; Robol forward,
5'-GAGGTAGCTATACTACGGGATGAC-3' and reverse, 5'-CAGATGTAGTAGCCGACATCAGAC-3'; U6 forward, 5'-CGCTTCGGCAGCACATATAC-3' and reverse, 5'-AAA ATATGGAACGCTTCACGA-3'.

Scratch wound assay. Cell migration ability was assessed using a scratch wound assay. Transfected cells were cultured in 6-well plates. When cells reached 90\% confluence, a scratch wound was created using a pipette tip. Wound edges were photographed with a Nikon Eclipse TE20000-U (Nikon, Tokyo, Japan) and scratch widths were analyzed using ImageJ software (NIH). Each assay was completed in triplicate.

Mouse model of OIR. Neonatal CB57BL/6J mice were obtained from the Animal Institute of Chinese Academy of Medical Sciences. Neovascularization was induced as described by Kong et al (27). Briefly, at postnatal day 7 (P7), CB57BL/6J mice were exposed to hypoxic conditions $\left(75 \% \mathrm{O}_{2}\right)$ for 5 days (P12) and then returned to room air in order to induce RNV.

The present study was approved by the Ethics Committee of Tianjin Eye Hospital (Heping, China). All animal experiments were conducted in accordance with the ARVO Statement for the Use of Animals in Ophthalmic and Vision Research and the Guide for the Care and Use of Laboratory Animals.

Intravitreal injection. Plasmids were packed using Lipofectamine 2000 (Invitrogen) in accordance with the manufacturer's instructions. Twelve-day-old mice were anesthetized by intraperitoneal injection of ketamine hydrochloride $(30 \mathrm{mg}$ / $\mathrm{kg}$ ). Each animal received intravitreous injections of $0.4 \mu \mathrm{l}$ $(2 \mu \mathrm{g}) \mathrm{pCDH}-\mathrm{CMV}-218$ in one eye and $0.4 \mu \mathrm{l}(2 \mu \mathrm{g})$ control plasmid [plasmid vector pCDH-CMV-MCS-EF1-Puro (Spiral Biotech, Inc.) without an insertion of miR-218] in the contralateral eye as the negative control. Intravitreal injections were performed at 11:00 a.m., and were administered to 1-mm posterior to the limbus of the eye using a $10-\mu 1$ Hamilton syringe fitted with a $32 \mathrm{G}$ needle.

Western blotting. Total proteins of cells and retinas were isolated and separated on 10\% SDS-PAGE gels (Bio-Rad Laboratories, Inc., Hercules, CA, USA). Western blotting was performed according to standard protocols. $\alpha$-tubulin was used as a loading control. The antibodies anti-Robol (ab7279), anti-Slit2 (ab7665), anti-Slit3 (ab11018) were from Abcam, and anti- $\alpha$-tubulin (T5168) was from Sigma-Aldrich (St. Louis, MO, USA). Bands were quantified using ImageJ software (NIH).

Fluorescein angiography. On P17, mice (n=6/group) were anesthetized and perfused with fluorescein via retro-orbital injection of $2.5 \mathrm{mg} / 50 \mu \mathrm{l}$ of FITC-dextran (Sigma-Aldrich), as previously described (28). Eyes were enucleated and fixed with $4 \%$ paraformaldehyde in PBS for $1 \mathrm{~h}$. Retinas were then separated from the eyecup. Four incisions were made, and the retina was flat-mounted on a gelatin-coated slide. The vasculature was then examined under a fluorescence microscope (Nikon Eclipse TE20000-U). Images were analyzed using Photoshop 8.0 software (Adobe Systems, San Jose, CA, USA). Neovascularization was calculated as a ratio: the number of pixels in neovascular area to the total number of pixels in the retina. 
A
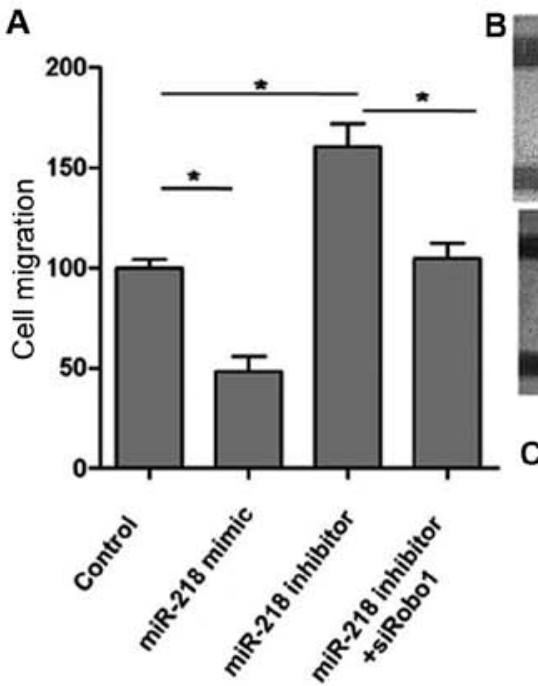

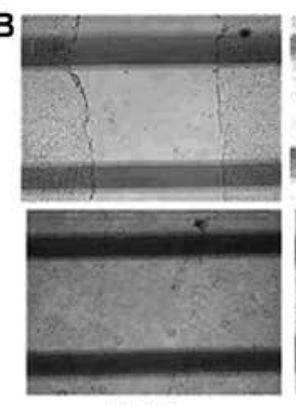

control

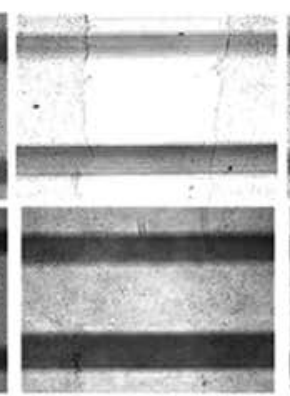

miR-218 mimic

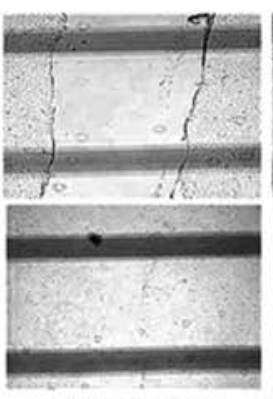

miR-218 inhibitor

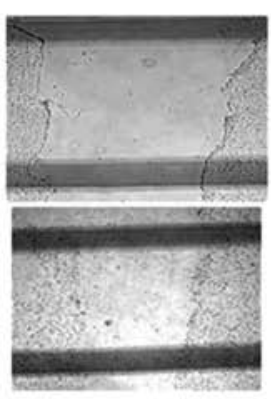

miR-218 inhibitor+siRobo1
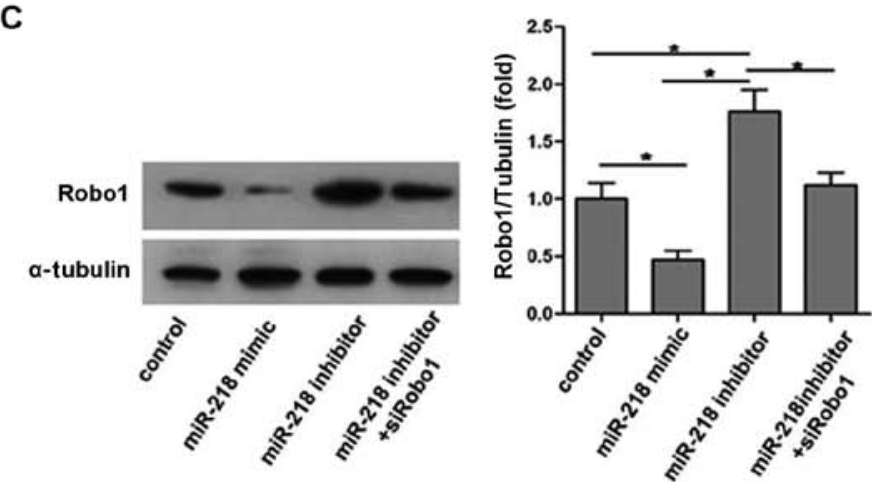

Figure 1. miR-218 inhibits endothelial cell (EC) migration by suppressing roundabout, axon guidance receptor, homolog 1 (Robo1) expression. (A) Quantification of cell migration. (B) Representative images of cell migration in the wounded area. (C) Robol expression was determined by western blotting and normalized to the internal control $\alpha$-tubulin. ${ }^{*} \mathrm{P}<0.05$.

Quantification of $R N V$. At p17, the eyes of the mice (n=6/group) were enucleated and fixed with $10 \%$ formaldehyde and embedded in paraffin. Sagittal sections (6- $\mu \mathrm{m}$-thick) were made through the cornea parallel to the optic nerve, and then they were stained with hematoxylin and eosin (H\&E) . The nuclei of vascular cells on the vitreal side of the retina were counted under a light microscope. Ten non-continuous sections from each eye were examined, and numbers of cells were averaged in each group. The average number of pre-retinal vascular nuclei was compared.

Statistical analysis. Data are expressed as the means \pm standard deviation (SD) and were analyzed using SPSS 11.5 (SPSS, Inc., Chicago, IL, USA). To compare multiple sets of data, one-way analysis of variance (ANOVA) was used. For paired data sets, the LSD t-test was used. Bivariate correlations were calculated by Spearman's rank correlation coefficients. A P-value $<0.05$ was considered to indicate a statistically significant difference.

\section{Results}

Inhibition of cell migration by miR-218 is mediated by Robol. The cell migration ability of each group is shown in Fig. 1: overexpression of miR-218 caused by miR-218 mimic significantly reduced endothelial cell (EC) migration, whereas inhibition of miR-218 expression using the miR-218 inhibitor markedly promoted EC migration (Fig. 1A and B).

Robol expression of each group is shown in Fig. 1C. The results indicate that upregulation of miR-218 expression not only reduced EC migration but also decreased Robol expression. Conversely, downregulation of miR-218 expression using the miR-218 inhibitor increased Robol expression and, as was noted previously, promoted EC mobility. However, as shown in Fig. 1A, miR-218 inhibitor did not promote the migratory ability of ECs after Robol knockdown by siRobo1. These observations suggest that miR-218 suppresses EC migration by inhibiting Robol expression.

miR-218 and Robol expression in RNV of mice with OIR. The expression level of miR-218 in the retinas of OIR mice was detected. As shown in Fig. 2A, RT-qPCR results demonstrated that the expression level of miR-218 was significantly decreased $(\mathrm{P}<0.05)$ in retinas of mice with OIR at P17. We then compared mRNA and protein expression levels of Robol in retinas of mice with OIR with those of the control mice. The mRNA and protein levels of Robol were markedly upregulated at P17 in mice with OIR (Fig. 2).

Role of miR-218 coding genes (miR-218-1 and miR-218-2) and corresponding host genes (Slit2 and Slit3). To determine which miR-218 coding gene is involved in downregulation of miR-218 in mice with OIR, we evaluated miR-218-1, miR-218-2, miR-218, Slit 2 mRNA and Slit 3 mRNA expression in retinas of mice with OIR by RT-qPCR. As shown in Fig. 3B-E, statistical analysis revealed that a positive correlation existed between miR-218-1 and Slit2, miR-218-2 and Slit3, as well as miR-218-1 and miR-218. There was no correlation, however, between miR-218-2 and miR-218. This meant that in the retinas of the mice with OIR, miR-218-1 and miR-218-2 were co-transcribed with their host gene respectively and that downregulation of miR-218 was caused by decreased expression of miR-218-1, not miR-218-2. When mRNA levels of Slit2 and Slit3 in retinas from control mice and mice with OIR were compared, mRNA expression of Slit2 in retinas of mice with OIR was 0.48 -fold less $(\mathrm{P}<0.05)$ than in the retinas of the normal control mice; 

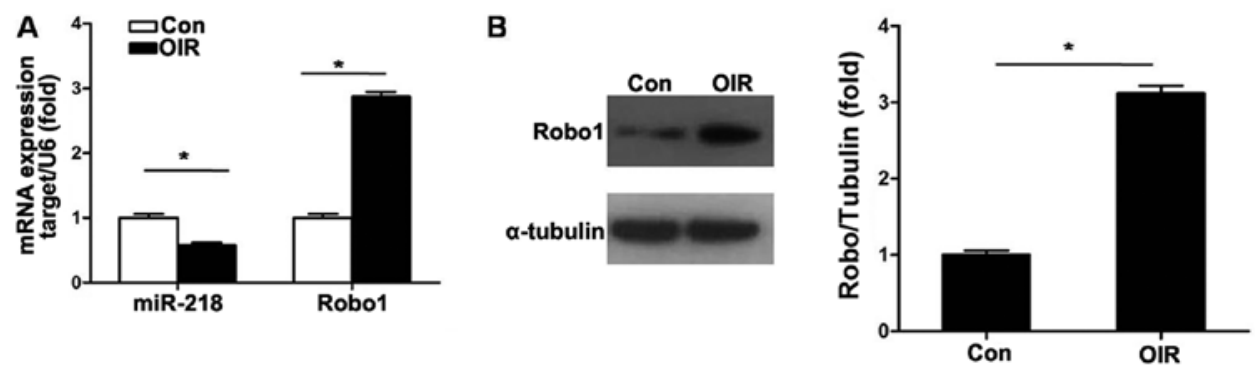

Figure 2. Identification of decreased miR-218 and increased roundabout, axon guidance receptor, homolog 1 (Robol) in retinas from the rat model of oxygeninduced retinopathy (OIR). (A) RT-qPCR showed that miR-218 was significantly decreased in the model of OIR compared to normal (control) mice (n=5). However, the expression of Robol mRNA was significantly increased $(n=6)$. (B) Western blot analysis demonstrated that Robol protein was highly expressed in retinas of mice with OIR compared to normal (control) mice. ${ }^{*} \mathrm{P}<0.05$.
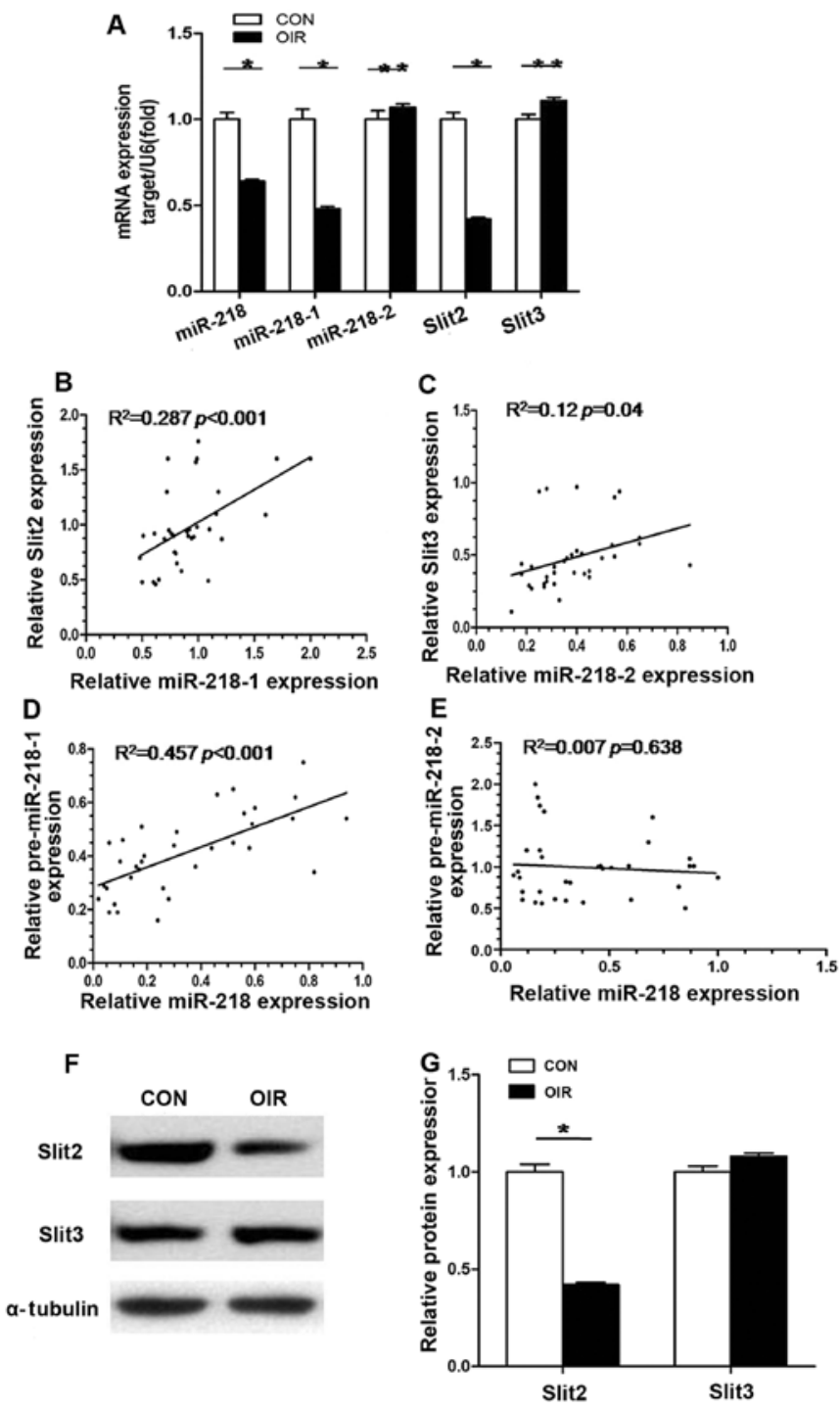

Figure 3. Expression of miR-218, miR-218-1, miR-218-2, Slit2 and Slit3 in retinas in mice with oxygen-induced retinopathy $(\mathrm{OIR})$ and control mice, as assessed by RT-qPCR. (A) Expression levels of miR-218, miR-218-1 and Slit2 were significantly decreased in OIR mice compared to normal mice. However, miR-218-2 and Slit3 were slightly elevated in OIR mice. (B and C) Positive correlations were observed between miR-218-1 and Slit2 as well as between miR-218-2 and Slit3. (D and E) Positive correlations were noted between miR-218-1 and miR-218. However, no significant positive correlation was noted between miR-218-2 and miR-218. (F) Representative images of western blotting of Slit 2 and Slit3 in mice with OIR and control mice. (G) Western blotting indicated that Slit2 protein expression significantly decreased $(\mathrm{P}<0.05)$. However, Slit3 protein expression was slightly elevated $(\mathrm{P}>0.05)$ in mice with OIR. ${ }^{*} \mathrm{P}<0.05,{ }^{* *} \mathrm{P}<0.01$.
mRNA expression of Slit3 in retinas from mice with OIR was 1.12-fold greater than in the control mice although this was not statistically significant $(\mathrm{P}>0.05)$ (Fig. 3A). Similar results were also observed in protein expression levels of Slit2 and Slit3 (Fig. 3F and G). The results suggest that decreased expression of miR-218-1 and its host gene Slit2 is involved in RNV in OIR.

miR-218 inhibits RNV through suppressing Robol expression. A plasmid pCDH-CMV-miR-218 expressing miR-218 and a negative control plasmid pCDH-CMV-mock expressing mock sequence were constructed. Intravitreal injection of plasmids was performed at P12.

At P17, we analyzed mRNA levels of miR-218 and Robol after intravitreal injection to verify whether plasmids reached the retina. RT-qPCR results showed that pCDH-CMV-miR-218 significantly increased the expression of miR-218 and reduced that of Robol in retinas (Fig. 4A). Western blotting indicated that intravitreal injection of pCDH-CMV-miR-218 significantly decreased the protein level of Robol in the retinas of mice with OIR (Fig. 4B). However, pCDH-CMV-mock had almost no effect on the expression of miR-218 and Robol.

To examine the effects of miR-218 on RNV, we evaluated the retinal vasculature at P17 by fluorescein angiography in the flat-mounted retinas. In retinas of normal mice we noted a mature capillary network that extended from the optic to the periphery (Fig. 5A1). The retinas of mice in the OIR and pCDH-CMV-mock groups displayed more neovascular tufts (Fig. 5A2 and A3). By contrast, the retinas of the pCDH-CMV-miR-218 group exhibited lesser neovascularization (Fig. A4). The RNV was quantified by measuring areas of new blood tufts in the whole mounted retina. The results showed that a large number of neovascular tufts appeared in the OIR group [neovascularization (NV), 38.9\% of whole retina] and pCDH-CMV-mock group (NV, $36.8 \%$ of whole retina). However, compared to the OIR group, the neovascularized area was significantly decreased in the pCDH-CMV-miR-218 group (NV, $18.6 \%$ of whole retina) (Fig. 5B). No neovascularization was observed in the normal group. These results revealed that miR-218 exerts an anti-neovascularizing effect on RNV in mice with OIR.

To further study the inhibitory effect of miR-218 on angiogenesis, histological analysis was performed. We counted the vascular cell nuclei which broke through the inner limiting membrane (ILM), a marked feature of OIR (29). There were no neovascular nuclei in the normal group (Fig. 6A1), and 

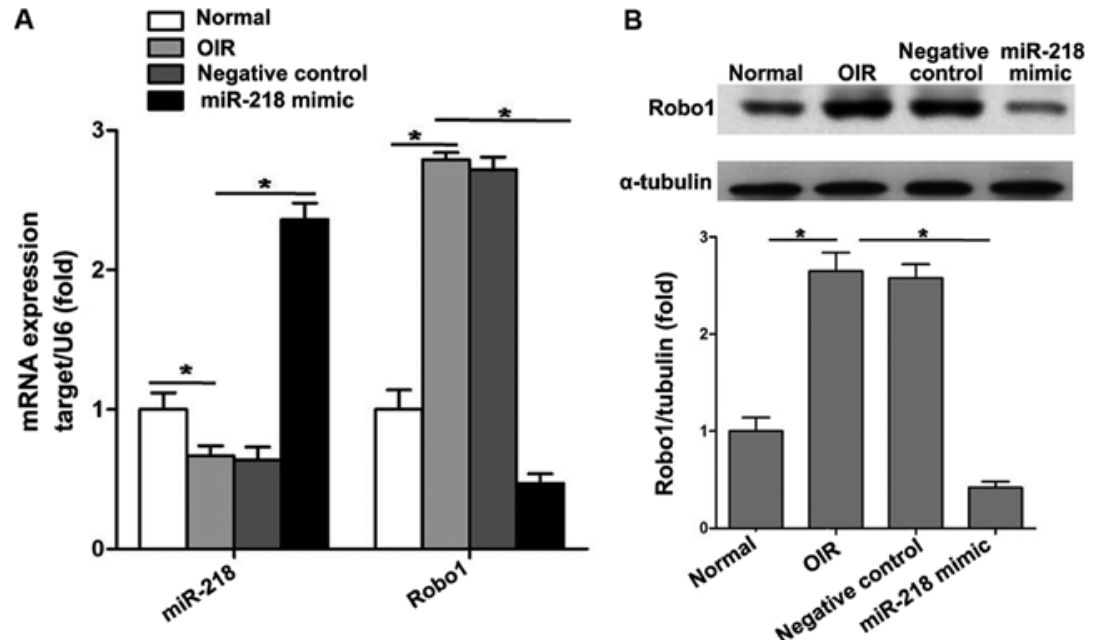

Figure 4. Downregulation of roundabout, axon guidance receptor, homolog 1 (Robo1) expression by intravitreal injection of miR-218 plasmid in mice with oxygeninduced retinopathy (OIR). (A) RT-qPCR results showed that relative Robol expression in the miR-218-transfected group was significantly decreased compared to the negative control group and OIR group. (B) Western blotting for Robol expression before and after miR-218 intravitreal injection. Statistical analysis of western blotting indicated that intravitreal injection of miR-218 effectively inhibited Robol protein expression in retinas in mice with OIR. $n=6$ mice/group. * $\mathrm{P}<0.05$.

the average number of vascular cell nuclei was significantly increased in the OIR group and pCDH-CMV-mock group, $61.48 \pm 6.92$ and 58.98 \pm 6.48 , respectively (Fig. 6A2 and A3). However, in the pCDH-CMV-miR-218 group we noted less neovascularization (Fig. 6A4) $(12.64 \pm 1.42$ pre-retinal cells/section), indicating that restoration of miR-218 played an inhibitory role in RNV in OIR (Fig. 6B).

Taken together, these results indicate that miR-218 inhibited retinal angiogenesis in OIR by suppressing Robol expression.

\section{Discussion}

Robo1, a member of the Robo receptor family, is involved in signaling in the nervous system (30). However, Robol plays a positive role in the migration of endothelial cells. Previous research using monkey choroidal retinal endothelial cells (31) and HUVECs (14) has demonstrated that decreased Robol markedly inhibited EC migration, and this conclusion was further confirmed using mouse retinal endothelial cell in this study.

In the present study, we noted that in vitro cell migration was markedly suppressed when the miR-218 mimic was transfected into ECs. Moreover, the expression of Robol decreased in cells. Additionally, a negative expression pattern was noted when miR-218-inhibitor was transfected into ECs: Robol expression increased with the decrease of miR-218 expression, and EC migration increased significantly. However, miR-218 inhibitor and siRobol had almost no effect on EC migration. Our results indicated that the inhibitory effect of miR-218 on EC migration was mediated by Robol.

The data presented in the present study indicate that mRNA and protein expression levels of Robol were significantly elevated in retinas of mice with OIR. This result was consistent with that of a previous study (31) and substantiated the potential role of Robol in RNV. Retinal vascular formation was also observed, suggesting that there was a significant positive correlation between Robol overexpression and RNV. Investigation of tumors indicated that Robol play a part in angiogenesis (14). Previous studies have suggested that Robol is involved in ocular neovascularization $(31,32)$. Genetic evidence provided by Rama et al supported a negative role for Robol in ocular pathological neovascularization (33). Our results also verified this point. In the present study, we found that retinal neovascularization and endothelial cells breaking into the inner nuclear layer in the retinas were both significantly reduced following the administration of pCDH-CMV-miR-218. Therefore, we suggest that downregulation of Robol significantly suppresses RNV.

In our study, a positive correlation between pre-miR-218 and their host gene was observed, demonstrating that miR-218-1 and miR-218-2 co-express with their host genes during RNV in OIR. Furtherore, a significant correlation between the expression of miR-218 and miR-218-1 was observed, indicating that the downregulation of miR-218-1 leads to the downregulation of miR-218. In addition, the expression of miR-218-1 and its host gene, Slit2 (not miR-218-2 and Slit3), were concomitantly downregulated during RNV in mice with OIR, indicating that miR-218-1 and Slit2 are involved in RNV by negatively regulating retinal angiogenesis.

As an intronic miRNA, miR-218 is involved in ligand/ receptor signaling. In brief, miR-218 is located in and is co-expressed with its host gene, Slit, whose receptor gene, Robol, is one of the targets of miR-218. Thus, Slit-miR-218-Robo signaling is established. Ligand/receptor signaling transduction mediated by intronic miRNAs has also demonstrated in other studies. miR-338, also an intronic miRNA, was shown to silence the target gene, which was antagonistic to its host gene, AATK (37). Tie et al (34) pointed out that miR-218 mediated Slit/Robo signaling, forming a negative feedback circuit, inhibiting gastric cancer metastasis. The Slit-miR-218-Robo regulatory network (26) was also found to be essential for the normal vascularization of the retina. In our study, RNV in mice with OIR was shown to be regulated by the Slit-miR-218-Robo axis. We thus speculated that the regulation of Slit-miR-218Robo signaling may provide a means of suppressing RNV by preventing the overactivation of ligand/receptor signaling.

In the mice with OIR, we noted that the expression of miR-218 significantly decreased, and the expression of Robol 
A
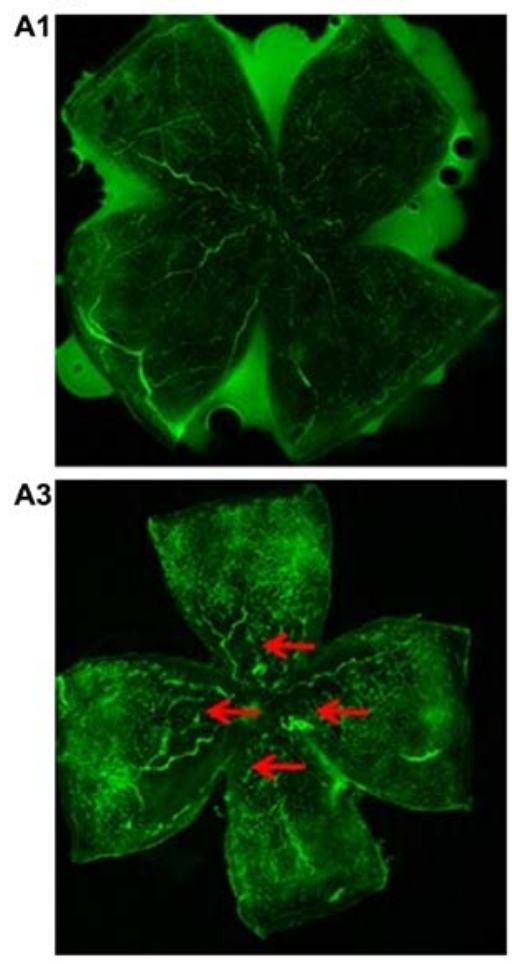

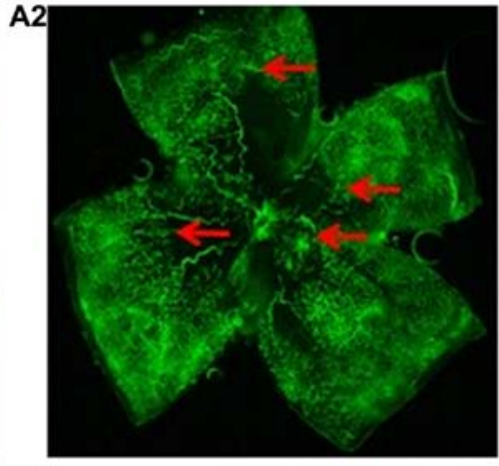

A4

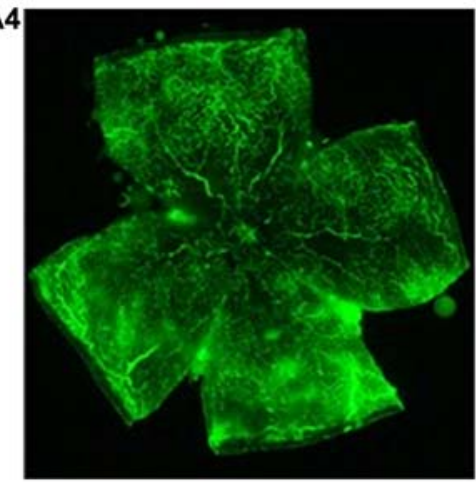

B

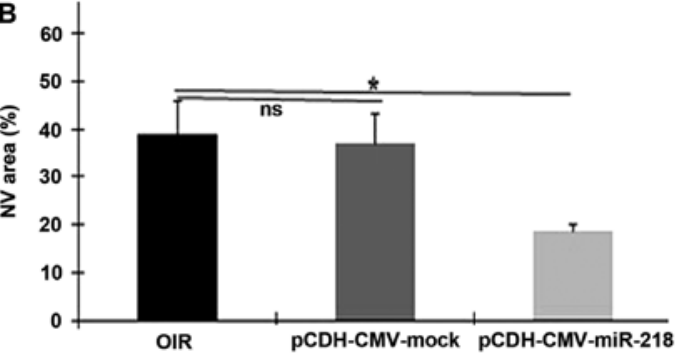

Figure 5. Representative retinal angiographs show that miR-218 effectively inhibits retinal neovascularization (RNV) in mice with oxygen-induced retinopathy (OIR) (magnification, x40). (A1) Retinal vessels in the normal mice. (A2 and A3) In the OIR group and pCDH-CMV-mock (negative control) group, tortuous and dilated retinal vessels were observed, and neovascular tufts were present at the junction between perfused and non-perfused regions (red arrows). (A4) After miR-218 injection, neovascular tuffs and non-perfused area were reduced. (B) Quantification of RNV from the group with OIR, pCDH-CMV-mock (negative control) group and miR-218-injected group (pCDH-CMV-miR-218). RNV was assessed using the ratio of the neovascular tuft area to total retinal area, and measured by image-analysis software. $\mathrm{n}=6$ mice/group. ${ }^{*} \mathrm{P}<0.05$.

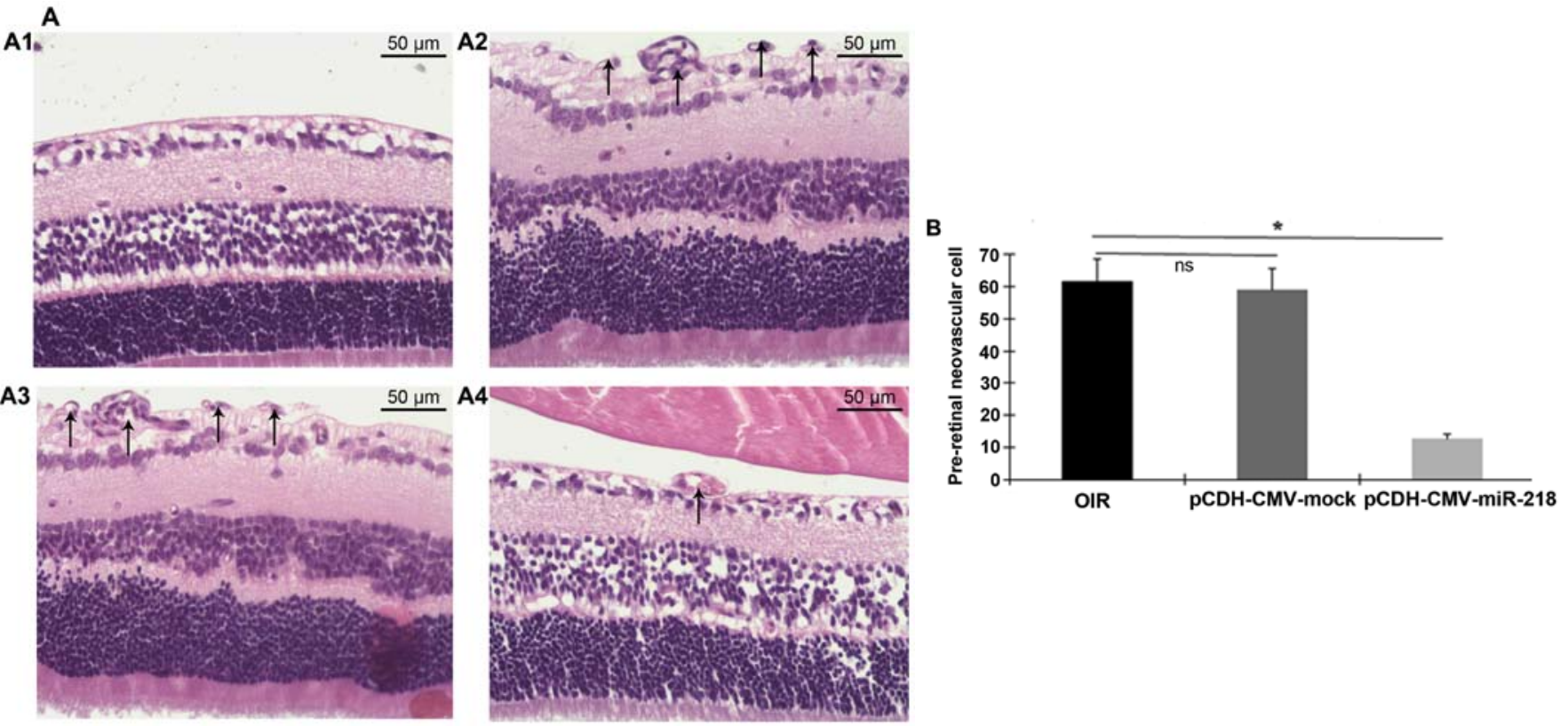

Figure 6. Hematoxylin and eosin (H\&E) staining of neovascularization in mouse retinas (magnification, $x 40)$. At P17, the retinas were fixed and stained. (A1) Normal group; (A2) OIR group; (A3) pCDH-CMV-mock group (negative control); (A4) miR-218 group. Arrows indicate pre-retinal vascular cells; (B) Vascular cell nuclei broke through the inner limiting membrane (ILM) (as indicated by arrows) and were counted in ten sagittal sections and averaged. The miR-218 group exhibited less neovascularization. $\mathrm{n}=6$ mice/group. " $\mathrm{P}<0.05 . \mathrm{NS}$, not significant.

increased. The restoration of miR-218 inhibited retinal angiogenesis, suggesting that miR-218 is an important regulator of RNV. Our results also suggest that miR-218 inhibits RNV through mediating the downregulation of Robol expression. 
In conclusion, the Slit-miR-218-Robo axis plays a role in retinal angiogenesis. Slit2 interacts with Robo1 to inhibit RNV mediated by miR-218. miR-218 may thus be a therapeutic candidate for the targeted treatment of RNV

\section{Acknowledgements}

This study was supported by the Natural Science Foundation of Tianjin City (no. 13JCYBJC22900).

\section{References}

1. Ishida S, Usui T, Yamashiro K, Kaji Y, Amano S, Ogura Y Hida T, Oguchi Y, Ambati J, Miller JW, et al: VEGF164-mediated inflammation is required for pathological, but not physiological, ischemia-induced retinal neovascularization. J Exp Med 198: 483-489, 2003

2. Avunduk AM, Cetinkaya K, Kapicioğlu Z and Kaya C: The effect of posterior vitreous detachment on the prognosis of branch retinal vein occlusion. Acta Ophthalmol Scand 75: 441-442, 1997.

3. Moravski CJ, Kelly DJ, Cooper ME, Gilbert RE, Bertram JF, Shahinfar S, Skinner SL and Wilkinson-Berka JL: Retinal neovascularization is prevented by blockade of the renin-angiotensin system. Hypertension 36: 1099-1104, 2000.

4. Campochiaro PA: Ocular neovascularization. J Mol Med Berl 91: 311-321, 2013.

5. Brose K, Bland KS, Wang KH, Arnott D, Henzel W, Goodman CS Tessier-Lavigne $\mathrm{M}$ and Kidd T: Slit proteins bind Robo receptors and have an evolutionarily conserved role in repulsive axon guidance. Cell 96: 795-806, 1999.

6. Dickson BJ: Molecular mechanisms of axon guidance. Science 298: 1959-1964, 2002.

7. Liu J, Zhang L, Wang D, Shen H, Jiang M, Mei P, Hayden PS, Sedor JR and Hu H: Congenital diaphragmatic hernia, kidney agenesis and cardiac defects associated with Slit3-deficiency in mice. Mech Dev 120: 1059-1070, 2003.

8. Cariboni A, Andrews WD, Memi F, Ypsilanti AR, Zelina P, Chedotal A and Parnavelas JG: Slit2 and Robo3 modulate the migration of GnRH-secreting neurons. Development 139: 3326-3331, 2012.

9. Kryczek I, Wei S, Keller E, Liu R and Zou W: Stroma-derived factor (SDF-1/CXCL12) and human tumor pathogenesis. Am J Physiol Cell Physiol 292: C987-C995, 2007.

10. Stella MC, Trusolino L and Comoglio PM: The Slit/Robo system suppresses hepatocyte growth factor-dependent invasion and morphogenesis. Mol Biol Cell 20: 642-657, 2009.

11. Bauer K, Dowejko A, Bosserhoff AK, Reichert TE and Bauer R Slit-2 facilitates interaction of P-cadherin with Robo-3 and inhibits cell migration in an oral squamous cell carcinoma cell line. Carcinogenesis 32: 935-943, 2011.

12. Schubert T, Denk AE, Ruedel A, Kaufmann S, Hustert E, Bastone P and Bosserhoff AK: Fragments of SLIT3 inhibit cellular migration. Int J Mol Med 30: 1133-1137, 2012.

13. Han X and Zhang MC: Potential anti-angiogenic role of Slit2 in corneal neovascularization. Exp Eye Res 90: 742-749, 2010.

14. Wang B, Xiao Y, Ding BB, Zhang N, Yuan X, Gui L, Qian KX, Duan S, Chen Z, Rao Y and Geng JG: Induction of tumor angiogenesis by Slit-Robo signaling and inhibition of cancer growth by blocking Robo activity. Cancer Cell 4: 19-29, 2003.

15. Zhou W, Yu W, Xie W, Huang L, Xu Y and Li X: The role of SLIT-ROBO signaling in proliferative diabetic retinopathy and retinal pigment epithelial cells. Mol Vis 17: 1526-1536, 2011.

16. Kim VN, Han J and Siomi MC: Biogenesis of small RNAs in animals. Nat Rev Mol Cell Biol 10: 126-139, 2009.

17. Kim YK and Kim VN: Processing of intronic microRNAs EMBO J 26: 775-783, 2007.
18. van Rooij E, Quiat D, Johnson BA, Sutherland LB, Qi X, Richardson JA, Kelm RJ Jr and Olson EN: A family of microRNAs encoded by myosin genes governs myosin expression and muscle performance. Dev Cell 17: 662-673, 2009.

19. Bartel DP: MicroRNAs: genomics, biogenesis, mechanism, and function. Cell 116: 281-297, 2004

20. Sayed D and Abdellatif M: MicroRNAs in development and disease. Physiol Rev 91: 827-887, 2011.

21. Kasinski AL and Slack FJ: Epigenetics and genetics. MicroRNAs en route to the clinic: progress in validating and targeting microRNAs for cancer therapy. Nat Rev Cancer 11: 849-864, 2011.

22. Fabian MR, Sonenberg N and Filipowicz W: Regulation of mRNA translation and stability by microRNAs. Annu Rev Biochem 79: 351-379, 2010.

23. Shen J, Yang X, Xie B, Chen Y, Swaim M, Hackett SF and Campochiaro PA: MicroRNAs regulate ocular neovascularization. Mol Ther 16: 1208-1216, 2008.

24. Bai Y, Bai X, Wang Z, Zhang X, Ruan C and Miao J: MicroRNA-126 inhibits ischemia-induced retinal neovascularization via regulating angiogenic growth factors. Exp Mol Pathol 91: 471-477, 2011 .

25. Chen N, Wang J, Hu Y, Cui B, Li W, Xu G, Liu L and Liu S: MicroRNA-410 reduces the expression of vascular endothelial growth factor and inhibits oxygen-induced retinal neovascularization. PLoS One 9: e95665, 2014.

26. Small EM, Sutherland LB, Rajagopalan KN, Wang S and Olson EN: MicroRNA-218 regulates vascular patterning by modulation of Slit-Robo signaling. Circ Res 107: 1336-1344, 2010.

27. Kong YC, Sun B, Zhao KX, Han M and Wang YC: Small interference RNA targeting vascular endothelial growth factor gene effectively attenuates retinal neovascularization in mice model. Chin Med J (Engl) 126: 1440-1444, 2013.

28. Li S, Li T, Luo Y, Yu H, Sun Y, Zhou H, Liang X, Huang J and Tang S: Retro-orbital injection of FITC-dextran is an effective and economical method for observing mouse retinal vessels. Mol Vis 17: 3566-3573, 2011.

29. Smith LE: Pathogenesis of retinopathy of prematurity. Acta Paediatr Suppl 91: 26-28, 2002.

30. Kidd T, Bland KS and Goodman CS: Slit is the midline repellent for the robo receptor in Drosophila. Cell 96: 785-794, 1999.

31. Huang L, Xu Y, Yu W, Li X, Liqun C, He X and Peiying H: Robol: a potential role in ocular angiogenesis. 34: 1019-1029, 2009.

32. Huang L, Yu W, Li X, Niu L, Li K and Li J: Robo1/robo4 different expression patterns in retinal development. Exp Eye Res 88: 583-588, 2009.

33. Rama N, Dubrac A, Mathivet T, Ní Chárthaigh RA, Genet G, Cristofaro B, Pibouin-Fragner L, Ma L, Eichmann A and Chédotal A: Slit2 signaling through Robo1 and Robo2 is required for retinal neovascularization. Nat Med 21: 483-491, 2015.

34. Tie J, Pan Y, Zhao L, Wu K, Liu J, Sun S, Guo X, Wang B, Gang Y,Zhang Y, et al: MiR-218 inhibits invasion and metastasis of gastric cancer by targeting the Robol receptor. PLoS Genet 6: e1000879, 2010

35. Fish JE, Wythe JD, Xiao T, Bruneau BG, Stainier DY, Srivastava D and Woo S: A Slit/miR-218/Robo regulatory loop is required during heart tube formation in zebrafish. Development 138: 1409-1419, 2011.

36. Alajez NM, Lenarduzzi M, Ito E, Hui AB, Shi W, Bruce J, Yue S, Huang SH, Xu W, Waldron J, et al: MiR-218 suppresses nasopharyngeal cancer progression through downregulation of survivin and the SLIT2-ROBO1 pathway. Cancer Res 71: 2381-2391, 2011.

37. Barik S: An intronic microRNA silences genes that are functionally antagonistic to its host gene. Nucleic Acids Res 36: 5232-5241, 2008. 